\title{
A New Plan for Toll Plaza
}

\author{
Yi Wang \\ School of North China Electric Power University, Baoding 71000, China
}

Keywords: Queuing Theory, VISSIM Simulation, Feedback Control, Analytic Hierarchy Process.

\begin{abstract}
The highway toll station is usually considered as the bottleneck of high way traffic flow. Therefore, it is essential to design a new scheme of toll plazas in highway, under the background of rapid traffic development and the emergency of self-driving. A reasonable design of toll plazas is to determine the shape, size, and merging pattern. We mainly consider designing the area following the toll barrier. we make evaluations about the performance of our new solution and normal solutions, considering the traffic capacity, construction cost and loss. By comparison, our new solution is better.
\end{abstract}

\section{Introduction}

With the gradual formation of the highway network, the drawbacks of tollbooths are increasingly apparent, which not only restricts the efficient operation of the entire highway transportation system, but also affect the overall image of the highway.

In recent years, science and technology improve dramatically. Exact-change (automated) tollbooths and electronic toll collection booths (such as electronic toll collection via a transponder in the vehicle) has been gradually promoted, which could greatly increase the capacity of toll collection system. In addition, the popularity of autonomous (self-driving) vehicles could enhance security.

\section{Models}

The toll plaza can be composed of two continuous queuing systems, one is the queuing system of toll collection (CQS) and the other is the queuing system of departure for lanes (DQS). we can know the service time of tollbooths obey the normal distribution. Therefore, taking queuing theory and statistical methods as the foundation, we develop a model.

According to the queuing theory, we can get the formula below [2]:

$$
\rho=\frac{\lambda E(x)}{B \mu}, \varphi_{b}=\frac{\sqrt{D(t)}}{E(t)}, E(t)=\frac{1}{\mu}
$$

The average queue length:

$$
L_{1}=\rho+\frac{\rho\left[\sigma_{x}^{2}+E^{2}(x)-E(x)\right]}{2(1-\rho) E(x)}+\frac{\rho^{2}\left(1+\varphi_{b}^{2}\right)}{2(1-\rho)}
$$

The average sojourn time:

$$
W_{1}=\frac{\rho\left[\sigma_{x}^{2}+E^{2}(x)+E(x) \varphi_{b}^{2}\right]}{2 B \mu(1-\rho) E(x)}+\frac{1}{B \mu}
$$

The total cost of CQS: $E_{1}=C_{S} B+C_{w} L_{1}$

\subsubsection{CQS}

When $B=1,2, \ldots, 10$, there are different $\mathrm{L}_{1}, \mathrm{~W}_{1}, \mathrm{E}_{1}$, their relationship are presented in Figure1: 


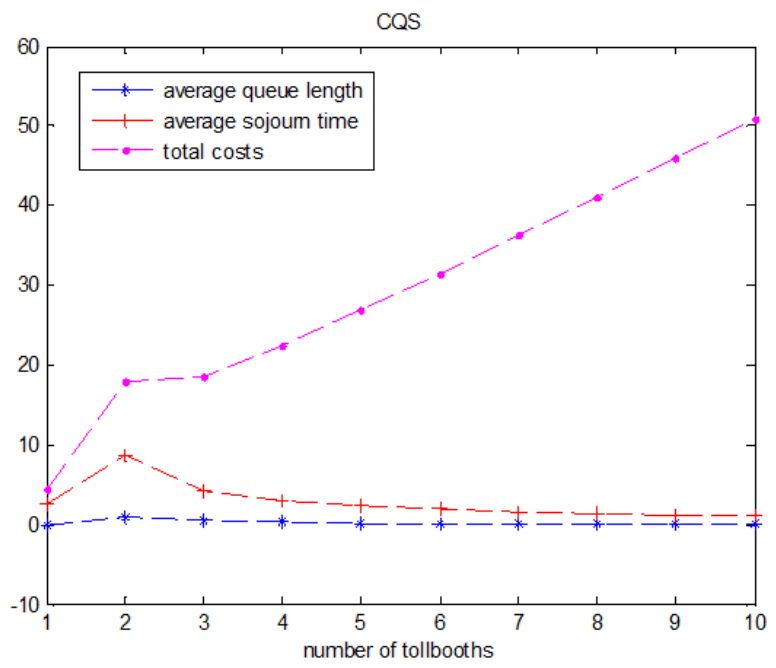

\subsubsection{DQS}

Figure 1: The influence of tollbooths numbers in $\mathrm{L}_{1}, \mathrm{~W}_{1}, \mathrm{E}_{1}$

According to the queuing theory, we can get

$$
\rho=\frac{\lambda E(y)}{l \mu}, \varphi_{b}=\frac{\sqrt{D(t)}}{E(t)}, E(t)=\frac{1}{\mu}
$$

The average queue length $L_{2}$ :

$$
L_{2}=\rho+\frac{\rho\left[\sigma_{y}{ }^{2}+E^{2}(y)-E(y)\right]}{2(1-\rho) E(y)}+\frac{\rho^{2}\left(1+\varphi_{b}{ }^{2}\right)}{2(1-\rho)}
$$

The average sojourn time $W_{2}$ :

$$
W_{2}=\frac{\rho\left[\sigma_{y}{ }^{2}+E^{2}(y)+E(y) \varphi_{b}{ }^{2}\right\rfloor}{2 l \mu(1-\rho) E(y)}+\frac{1}{l \mu}
$$

The total cost of DQS:

$$
E_{2}=C_{w} L_{2}
$$

The same steps with (1), When $l=1,2, \ldots, 10$, there are different $\mathrm{L}_{2}, \mathrm{~W}_{2}, \mathrm{E}_{2}$, their relationship is presented in Figure2:

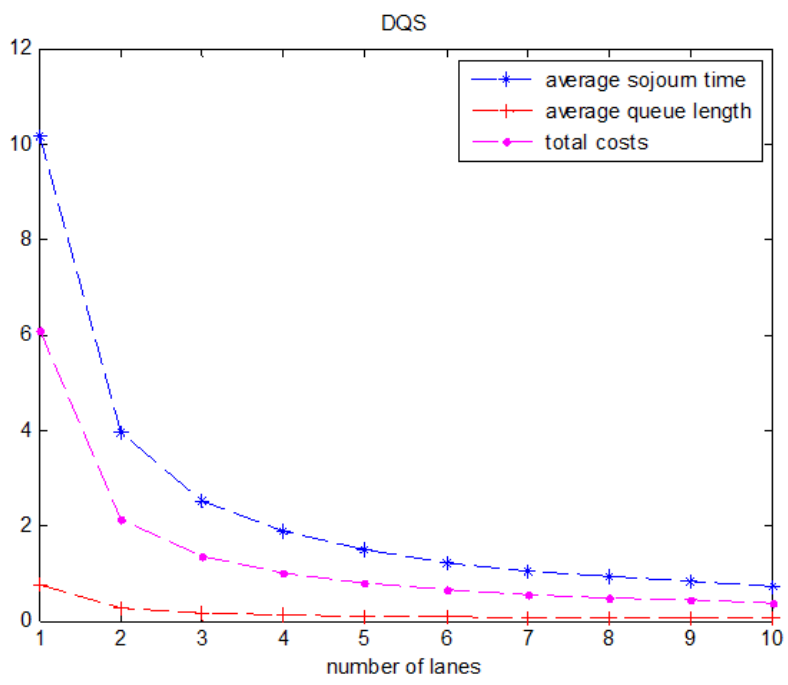

Figure 2: The influence of lanes number in $\mathrm{L}_{2} . \mathrm{W}_{2} . \mathrm{E}_{2}$

\subsubsection{Optimized model of numbers of lanes and tollbooths}

From (2),(3),(5),(6), we have the junction:

The total average sojourn time $W: W=W_{1}+W_{2}$

The total mathematics expectation of cost: $\quad E=E_{1}+E_{2}$ 
The best result is the shortest total average sojourn time and the least total costs, but they are contradictory. Therefore, we employ analytic hierarchy process method to optimize the objective function. On account of the difference of units of $W$ and $E$, make some changes about the function:

$$
\begin{aligned}
& \min =\alpha C_{w} W+\varphi E \\
& \text { s.t. }\left\{\begin{array}{l}
\rho_{1}, \rho_{2}<1 \\
\rho_{1}>\rho_{10}>0, \rho_{2}>\rho_{20}>0 \\
B \geq l \\
\alpha+\varphi=1, \alpha>\varphi
\end{array}\right.
\end{aligned}
$$

\subsection{Model of combined toll collection}

Model above is built with the assumption that all tollbooths are conventional (human-staffed) tollbooths (CTC). With the development of science and technology, exact-change (automated) tollbooths (ATC) and electronic toll collection booths(ETC) are introduced into highway toll system to decrease the average sojourn time.

Basing on the efficiency indexes of $M|N| 1$ model and considering nonelectronic toll collection $(\mathrm{CTC}+\mathrm{ATC}=\mathrm{MTC})$ and electronic toll collection(ETC), we get:

$$
\begin{aligned}
& N_{M}=\frac{(1-\beta)^{2} \lambda^{2}}{C_{M} \mu_{M}{ }^{2}\left[1-\frac{(1-\beta) \lambda}{C \mu_{M}}\right]}, N_{E}=\frac{\beta^{2} \lambda^{2}}{C_{E} \mu_{E}{ }^{2}\left[1-\frac{\beta \lambda}{C_{E} \mu_{E}}\right]} \\
& P_{M}=\beta\left(1-\rho_{M}\right), P_{E}=(1-\beta)\left(1-\rho_{E}\right)
\end{aligned}
$$

The total loss of costs during unit time is defined as: $S=N_{E} C_{w E}+N_{M} C_{w M}+P_{E} C_{f E}+P_{M} C_{f M}$

Putting these together, we get: $\left\{\begin{array}{l}\operatorname{Min}(S)=S\left(C_{E}, C_{M}\right) \\ C_{E}, C_{M} \in N^{+}\end{array}\right.$

\subsection{Cellular automaton and simulation model of tandem tollbooths [3]}

Parallel tollbooths, taking Poisson distribution as the foundation, has some shortages in time and space complexity, cost, reliability and other aspects. Concerning these shortcomings, cellular automaton and simulation model ${ }^{[1]}$ of tandem tollbooths is built to reduce effectively traffic congestion.

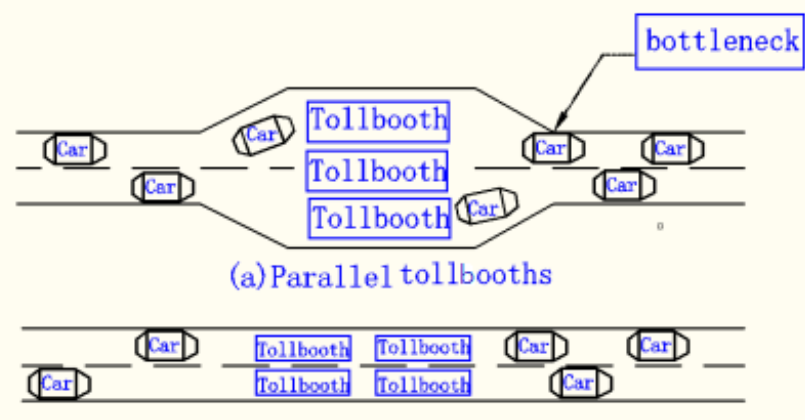

(b) Tandem tollbooths

Figure 3: Parallel tollbooths and tandemtollbooths

\section{Simulation model of traffic}

On account of the bottleneck phenomenon of merge area at highway toll station, we introduce an automatic feedback control system into the design to solve the problem of traffic congestion and avoid the occurrence of accident. Then, use VISSIM to develop a macroscopic simulation model to simulate the traffic situation of the peak hours and optimize the design of toll plazas. 
-The mechanism of automatic feedback control system

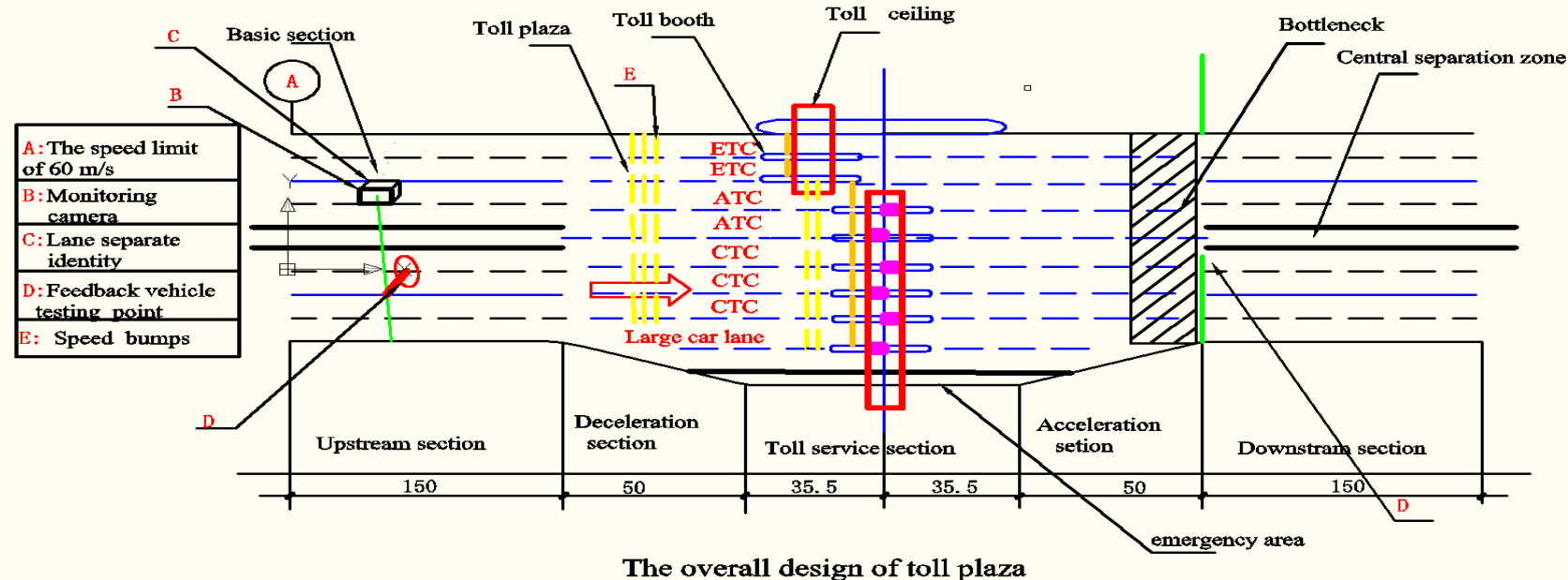

Figure 4: The overall design of toll plazas

\section{- The computing method of total area}

Based on the principle of the lowest costs and delay time, we get the best design proportion of the number of lanes and tollbooths with the relationship of them discussed previous, Then, according to the length of time, we obtain the weighted average queuing length of different cars. In order to reduce the cost of construction, the vehicle turning angle is too fierce, which result in the waste of cost, so use circular bead. Take the weighted average length of the queue as the length of corresponding area ,multiplied by the width of the lane to get the area of each lane, then sum up each area to get the total area.

\section{- A macroscopic simulation model}

Use VISSIM to develop a macroscopic simulation model to simulate the traffic situation at the toll station, as presented in figure 5:

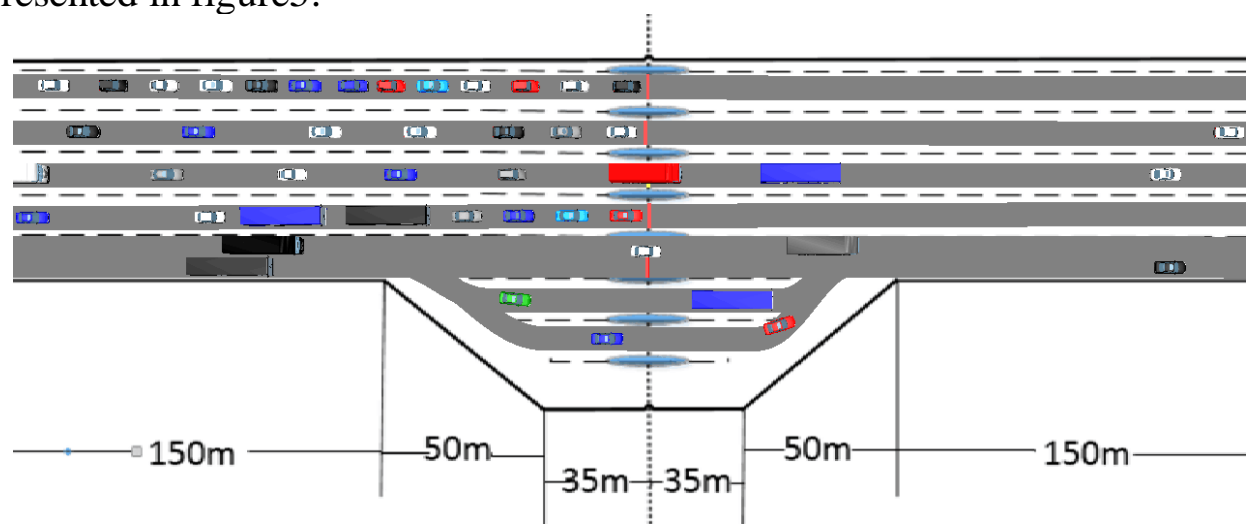

Figure 5: Macroscopic traffic simulation

\section{Considering autonomous (self-driving) vehicle}

Self-driving is a collaborative driving. On the premise of traffic safety and efficiency, make full use of road condition. Road cycling team not only has characters of fast speed and small spacing, but also according to different road conditions, through coordination and cooperation among the teams, on the one hand, simplify the complexity of traffic control and management system, effectively mitigate traffic congestion; on the other hand, reduce traffic accidents caused by human factors, ensure the traffic security and reduce environmental pollution on the basis of energy conservation. Autonomous (self-driving) vehicles have preferential choice of lanes, which fundamentally influence some important factors such as the number of arrival vehicle, throughput and sojourn time, thus affect the cost and make the cost is higher than the most suitable value. 


\section{References}

[1] Brian Camley. Bradley Klingenberg. Pascal Getreuer. For Whom the Booth Tolls.[2005]

[2] Min Sha. Haiyan Chen. Xuan Li. The design model of tollbooths.[2010]

[3] CHEN Haijun, WU Wei, DU Ronghua. Simulation of traffic capacity at highway serial toll station. Computer Engineering and Application,[2012] 